\title{
Aceros para embutido profundo: fabricación, caracterización, microestructura y texturas
}

\author{
K. Sipos*, J. Martínez*, N. Burgos* y H. Pesenti**
}

\begin{abstract}
Resumen El conocimiento de las características de formabilidad de las chapas metálicas es de gran interés tecnológico para la industria del conformado de planchas de acero. En el presente trabajo se muestra cómo se evalúan las chapas en su aptitud a la embutición a través de variados parámetros de propiedades mecánicas, y cómo éstas se relacionan con su fabricación en la industria siderúrgica. Durante este proceso, el conocimiento de los fenómenos metalúrgicos en las distintas etapas permiten el correcto control de las texturas cristalográficas, las cuales, finalmente, permiten el desarrollo de buenos índices de anisotropía normal y planar. Se muestra, a través de ejemplos industriales, la relación entre los parámetros operativos y los resultados obtenidos, ilustrándose éstos no solo a través de valores de los índices de anisotropía sino que también a través de las texturas obtenidas. La caracterización del producto a través de las curvas de deformación límite permite a las empresas que producen piezas de diseños particulares evaluar sus diseños y la conveniencia o no del uso de diferentes tipos de aceros para su fabricación. Se muestra como ejemplo el caso de cubiertas de enceradoras.
\end{abstract}

Palabras clave Aceros. Embutición. Formabilidad. Anisotropía. Texturas.

\section{Steels for deep drawing: manufacture, characterization, microstructure and textures}

\begin{abstract}
The knowledge of the characteristics of formability of steel sheets is of high technological interest in the deep drawing industry. In this paper we show how steel sheets are evaluated for their formability through various parameters of their mechanical properties, and how they are related to their manufacture at the steel plant. During this process the knowledge of the different metallurgical phenomena in the various steps allow the correct control of the cristalographical textures, which finally permit the development of good normal and planar anisotropy indexes. Industrial examples show the relationship between the operative parameters and the obtained results, illustrated not only through the values of the anisotropy indexes but also through the obtained textures. The characterization of the product through the forming limit curves permits to evaluate different product designs and the use of different types of steels for their manufacture. An example of a polishing machine cover is shown.
\end{abstract}

Keywords Steels. Deep Drawing. Formability. Anisotropy. Textures.

\section{INTRODUCCIÓN}

La elaboración de módulos embutidos de acero, es una de las operaciones más importantes en la industria de manufactura de producción en serie. Este procedimiento logra la obtención de piezas de gran calidad y homogeneidad. Hoy en día, la chapa estampada es utilizada en una amplia gama de aplicaciones industriales, como en guardafangos de au- tomóviles, piezas de aeroplanos, cubiertas de enceradoras, ruedas de carretillas, etc. En la industria, la chapa embutida ha ido sustituyendo lentamente a la soldadura, los tornillos y pernos para unir partes, de manera de tener una única pieza en vez de un conjunto complejo de partes. La simplicidad, la ligereza, la mayor resistencia mecánica y, principalmente, los reducidos costos de operación, son las ventajas del proceso de embutición. 
El buen resultado de la embutición depende no solo del cuidado con que se efectúa el proceso, sino también de las características del acero utilizado. La capacidad de deformación de la plancha es factor preponderante para que no aparezcan fallas en la superficie.

Por ello, la fabricación de láminas de acero para embutido requiere de un largo proceso de operaciones, siendo las más importantes la laminación en caliente, la laminación en frío y el recocido. Durante la elaboración de las láminas existen límites que condicionan los procesos metalúrgicos, tales como la deformación plástica y la recristalización del acero. Estas, fijan las propiedades mecánicas del material. Al dominar las variables de diseño y metalúrgicas se logran estructuras metalográficas adecuadas y una textura cristalográfica en una orientación preferencial que favorezca la deformación en el plano de la chapa. En 1949 se comenzaron a entender las relaciones de embutibilidad y ciertas características microestructurales del acero, sin entender todavía la estrecha relación entre los parámetros de fabricación del acero en sus diversas etapas de manufactura y los fenómenos metalúrgicos involucrados ${ }^{[1]}$. Así los autores indican: "La formación de granos recristalizados gruesos y alargados parece pues suceder debido a algún factor que reduce el número de núcleos efectivos de recristalización y que también limita el crecimiento de grano. La naturaleza precisa de esta influencia limitante no ha sido establecida".

\section{DESARROLLO EXPERIMENTAL}

$\mathrm{El}$ acero obtenido por proceso BOF y Horno $\mathrm{Cu}$ chara fue colado en planchones de $156 \mathrm{~mm}$ de espesor con la composición química que se indica en la tabla I.

La figura 1 representa en forma esquemática el proceso de elaboración del material y las variables más importantes a considerar durante la laminación ${ }^{[2]}$.

El fenómeno que establece las características de embutibilidad es la textura cristalina que se obtiene al final de la etapa de fabricación de la chapa de

Tabla I. Composición química del acero calidad embutido profundo

Table I. Chemical composition of deep drawing steel

\begin{tabular}{ccccccc}
\hline$\%$ C & $\%$ Mn & $\%$ P & \% S & \% Si & $\%$ Al & $\%$ V \\
\hline 0,047 & 0,250 & 0,009 & 0,005 & 0,019 & 0,022 & 0,017 \\
\hline
\end{tabular}

Rev. Metal. Madrid Vol. Extr. (2005) 58-63

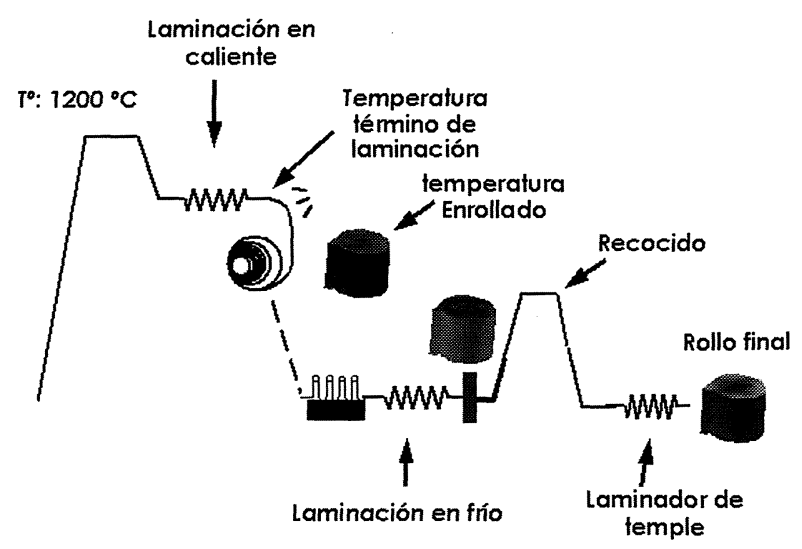

Figura 1. Esquema de producción del acero calidad embutido profundo.

Figure 1. Schematic diagram of manufacture of deep drawing steel.

acero. Una estructura cristalina preferencial de textura $(111)<110>$ permite una deformación biaxial de la chapa sin afectar en forma importante el espesor. La posibilidad de obtener esta textura es producto de la recristalización de la estructura metalográfica, obtenida por una fuerte deformación en frío, guiada por la acción restrictiva de la presencia de precipitados de nitruros de aluminio en cantidad y tamaño adecuados. El control de estos últimos se realiza a través del manejo de la historia térmica del acero, en particular el tiempo del material a temperatura de nucleación de los nitruros y su crecimiento posterior. Así, si el recocido es batch, debe evitarse la presencia de los nitruros previamente al recocido, ya que es durante el largo calentamiento de la bobina en el horno cuando estos precipitados aparecen y crecen de manera de controlar adecuadamente la recristalización. Una presencia previa de estos nitruros se traduciría en una coalescencia de los nitruros presentes y su crecimiento, de modo que se vuelven absolutamente inadecuados para lograr un control de la recristalización que permita obtener una textura $(111)<110>$.

La tabla II resume los datos de la laminación en caliente y laminación en frío.

Las rollos obtenidos fueron recocidos en proceso batch sometiéndolos a un calentamiento de $30^{\circ} \mathrm{C}$ por hora hasta llegar a los $700{ }^{\circ} \mathrm{C}$ en el punto mas frío, temperatura que se mantuvo durante $12 \mathrm{~h}$ para lograr una recristalización completa. De esta forma se logró obtener granos recristalizados alargados, del tipo denominado "panqueque", y una textura cristalográfica $(111)<110>$.

El proceso terminó con un enfriamiento controlado hasta los $160^{\circ} \mathrm{C}$, para terminar enfriando los 
Tabla II. Datos de laminación en caliente y en frío

Table II. Hot and cold rolling data

\begin{tabular}{ccccccc}
\hline & \multicolumn{2}{c}{ LAMINACIÓN EN CALIENTE } & \multicolumn{3}{c}{ LAMINACIÓN EN FRIO } \\
\hline $\begin{array}{c}\text { T }\left({ }^{\circ} \mathrm{C}\right) \text { fin } \\
\text { laminación }\end{array}$ & $\begin{array}{c}\text { \% reducción } \\
\text { último marco }\end{array}$ & $\begin{array}{c}\text { Espesor } \\
\text { salida }(\mathrm{mm})\end{array}$ & $\begin{array}{c}\mathrm{T}\left({ }^{\circ} \mathrm{C}\right) \\
\text { enrollado }\end{array}$ & $\begin{array}{c}\text { Espesor } \\
\text { entrada }(\mathrm{mm})\end{array}$ & $\begin{array}{c}\text { \% reducción } \\
\text { laminación }\end{array}$ & $\begin{array}{c}\text { Espesor } \\
\text { final }(\mathrm{mm})\end{array}$ \\
\hline 900 & 8,0 & 4,5 & 563 & 4,5 & 69 & 1,4 \\
900 & 12,5 & 3,8 & 558 & 3,8 & 74 & 1,0 \\
900 & 13,6 & 3,4 & 555 & 3,4 & 76 & 0,8 \\
\hline
\end{tabular}

rollos hasta la temperatura ambiente al aire calmo. Después del paso por el laminador de temple se tomaron muestras en la cabeza y en la cola de los rollos para su evaluación, tanto en el aspecto metalográfico como de sus propiedades mecánicas.

\section{PROPIEDADES CRISTALOGRAFICAS, META- LOGRAFICAS Y MECANICAS}

\subsection{Propiedades cristalográficas y metalo- gráficas}

Las propiedades metalográficas se evaluaron en los distintos pasos del proceso mediante la textura cristalográfica obtenida y también por la microestructura.

La figura 2 ilustra las texturas cristalográficas obtenidas en los distintos pasos del proceso. La evolución de éstas pasa por la desaparición paulatina de las texturas tipo $(001)<110>$ típicas del pro- ducto laminado en caliente y el refuerzo cada vez mas intenso de la fibra gamma con texturas del tipo $(111)<110>$.

Las micrografías de la estructura metalográfica se ilustran en la figura 3, mostrando una clara estructura equiaxial del producto laminado en caliente, fuertemente deformada en el producto laminado en frío y recristalizada alargada tipo "panqueque" en el producto después del recocido.

\subsection{Propiedades mecánicas}

Las características mecánicas del acero y sus propiedades de embutibilidad se midieron de las muestras tomadas del producto final. Para evaluar la anisotropía del material se efectuaron ensayos de tracción a distintos ángulos a la dirección de laminación. De los resultados obtenidos se evaluaron los índices " $\mathrm{r}$ " y "delta-r", como también se evaluó la capacidad de deformación plástica del

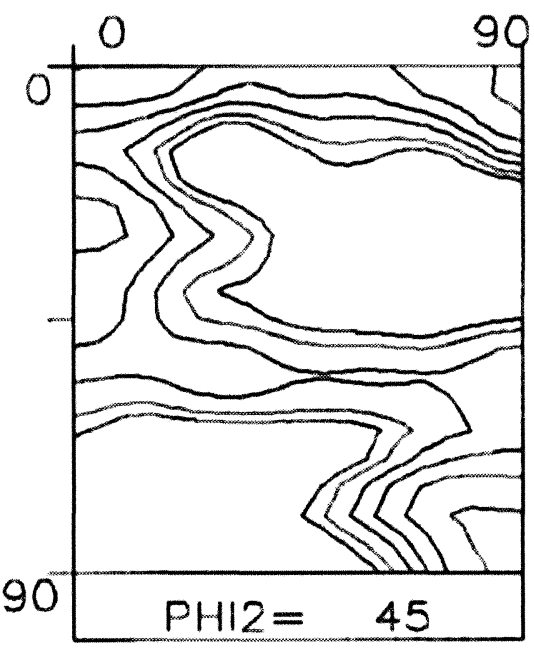

Producto laminado en caliente

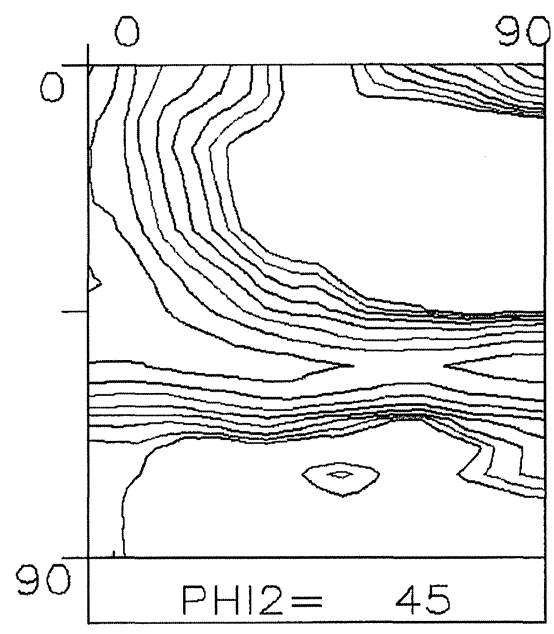

Producto laminado en frío

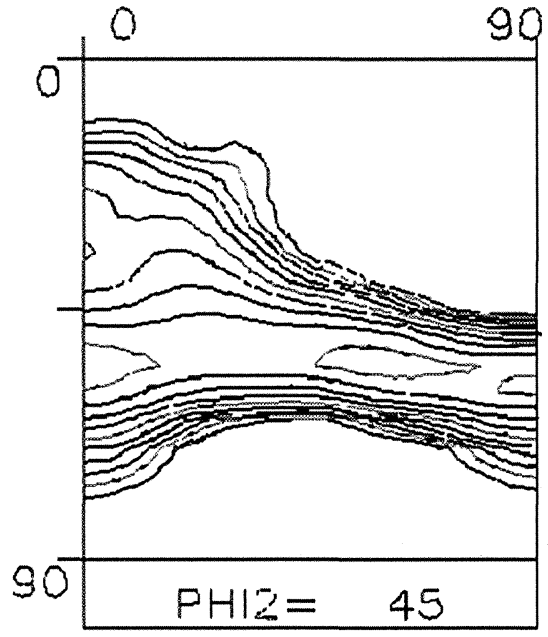

Producto después del recocido

Figura 2. Evolución de la textura a lo largo del proceso de laminado.

Figure 2. Texture development throughout the rolling process.

60 


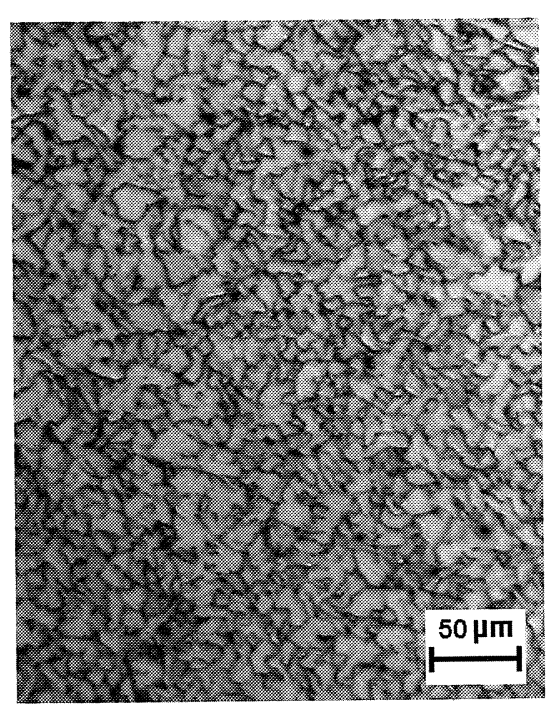

Producto laminado en caliente

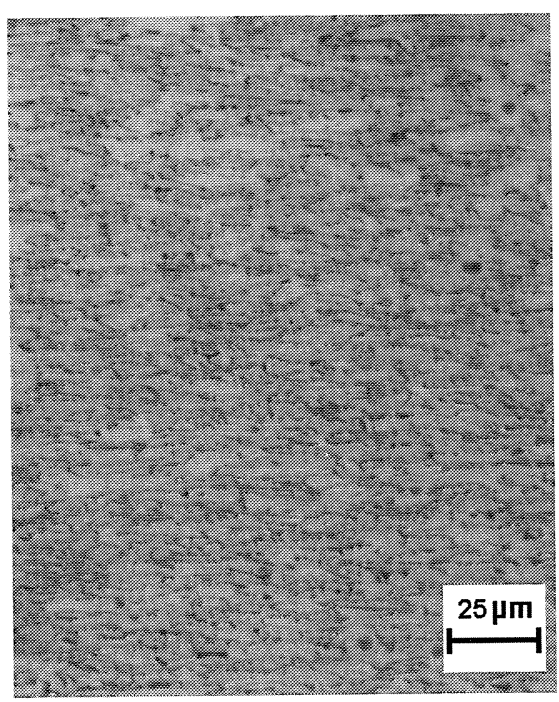

Producto laminado

en frío

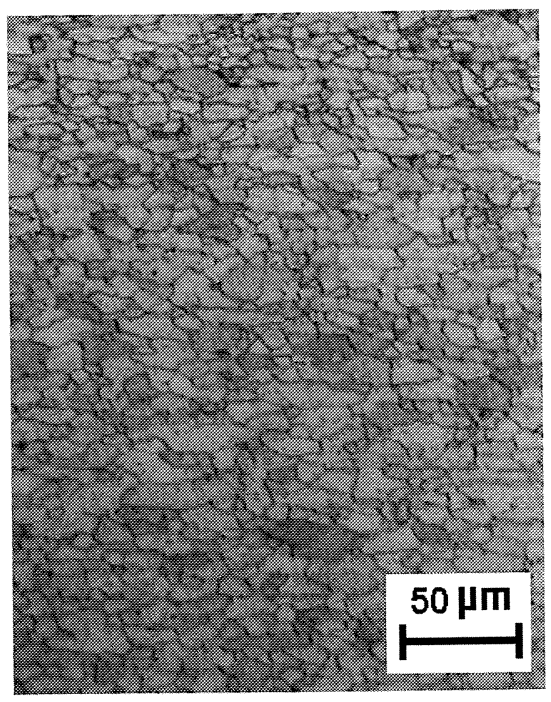

Producto después del recocido

Figura 3. Evolución de la microestructura a lo largo del proceso de laminado.

Figure 3. Texture development throughout the rolling process.

material midiendo el porcentaje de elongación y el coeficiente de endurecimiento "n".

La figura 4 ilustra los resultados de los ensayos de tracción, mostrando una baja en las propiedades en la dirección de $45^{\circ}$ respecto de la dirección de laminación.

La tabla III entrega la variación del coeficiente de endurecimiento " $n$ " en función del ángulo del ensayo de tracción referente a la dirección de laminación. También se muestran los valores de anisotropía " $\mathrm{r}$ ", definido como la relación de las deformaciones según el ancho y el espesor de una probeta de tracción uniaxial. El método de medición es difí- cil y de gran dispersión. La determinación de estos mismos índices por el método magnetoestrictivo, mas simple de efectuar, presenta resultados similares, y es muy consistente y repetitivo.

Evaluando los índices de anisotropía normal "r" y planar "delta-r" a partir de estos resultados se calculan valores de 1,5 y 0,35 , respectivamente. $\mathrm{La}$ mayor dificultad de medición de estos índices, a partir de las probetas de tracción y la buena homologación de resultados, sugiere que la medición por medios magnetoestrictivos es mas conveniente. Una evaluación estadística posterior muestra que los procesos de producción indicados mantienen

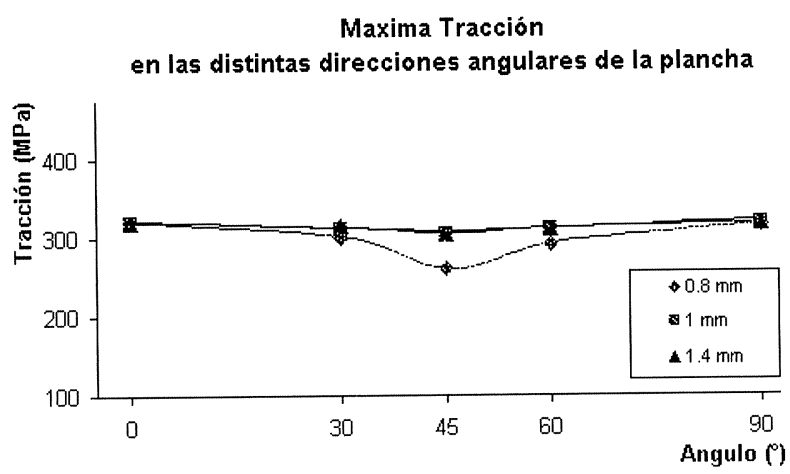

Resistencia a la tracción

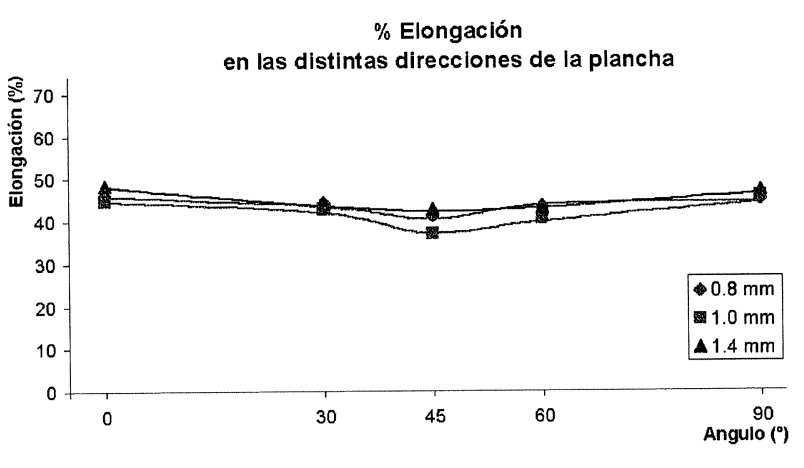

Propiedades de elongación

Figura 4. Propiedades mecánicas en función del ángulo hacia la dirección de laminación.

Figure 4. Mechanical properties depending on the angle to the rolling direction.

Rev. Metal. Madrid Vol. Extr. (2005) 58-63 
Tabla III. Coeficientes " $n$ " $y$ " $r$ " en las distintas direcciones respecto a la laminación

Table III. Indexes " $n$ " and " $r$ " depending on the angle to the rolling direction

\begin{tabular}{|c|c|c|c|c|c|c|c|c|c|c|c|}
\hline \multirow[b]{2}{*}{$\begin{array}{l}\text { Espesor } \\
(\mathrm{mm})\end{array}$} & \multicolumn{5}{|c|}{ Coeficiente $n$} & \multicolumn{6}{|c|}{ Anisotropía } \\
\hline & $0^{\circ}$ & $30^{\circ}$ & $45^{\circ}$ & $60^{\circ}$ & $90^{\circ}$ & Muestra & $0^{\circ}$ & $45^{\circ}$ & $90^{\circ}$ & $\begin{array}{c}r \\
\text { total }\end{array}$ & $\begin{array}{c}\mathbf{r} \\
\text { magneto }\end{array}$ \\
\hline 0,8 & 0,27 & 0,23 & 0,26 & 0,22 & 0,29 & $479-2$ & 1,7 & 1,2 & 2,0 & 1,5 & 1,5 \\
\hline 1,0 & 0,19 & 0,19 & 0,20 & 0,17 & 0,21 & $545-2$ & 1,4 & 1,2 & 1,6 & 1,4 & 1,5 \\
\hline 1,4 & 0,19 & 0,19 & 0,20 & 0,18 & 0,20 & $578-2$ & 1,4 & 1,2 & 1,9 & 1,4 & 1,4 \\
\hline
\end{tabular}

los valores de los índices de anisotropía normal "r" y planar "delta-r" en forma repetitiva, en valores sobre 1,5 , los primeros, y bajo 0,5 , los segundos, tal como sugiere la norma.

Para evaluar la aptitud del material para su embutibilidad se efectuaron ensayos que simulan procesos industriales: los ensayos de taza (Erichsen y similares) y las curvas límite de formabilidad. Estos ensayos son medios prácticos para entregar a los usuarios antecedentes de la calidad del material frente a esfuerzos similares a los que se presentan en condiciones reales de trabajo. De estos métodos, el mas práctico, por su aplicación directa en la industria, es el diagrama límite de deformación ${ }^{[3]}$, también llamado curvas límite de formabilidad, cuyo concepto se ilustra en la figura 5 . Este proceso evalúa las deformaciones máximas en el plano de la chapa, y las perpendiculares a éstas, en condiciones de exigencias de esfuerzo en el plano de la chapa. Utilizando estas herramientas se trazaron

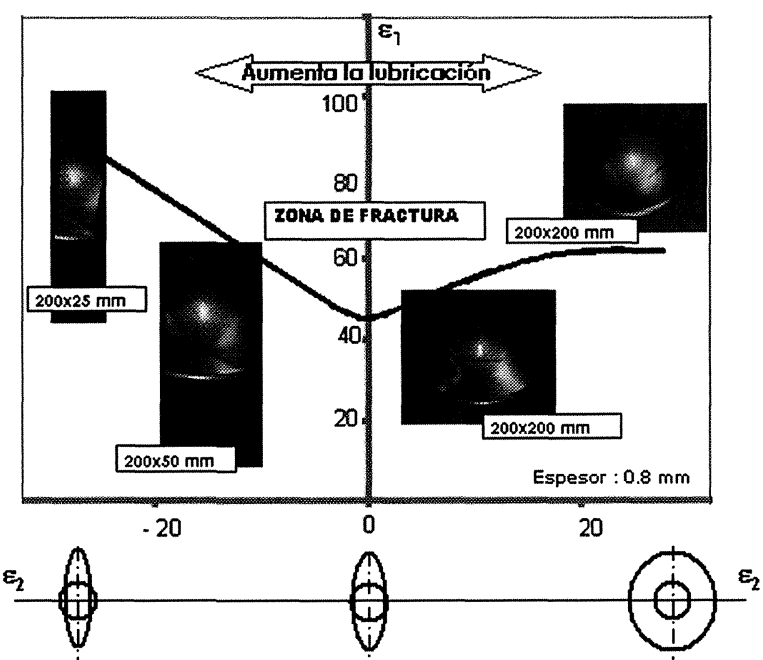

Figura 5. Diagrama límite de deformación.

Figure 5. Forming limit curve.

62 las curvas límite de formabilidad, una de las cuales se muestra en la figura 6 .

\section{CONCLUSIONES}

La utilización industrial de estas curvas pasa por efectuar una evaluación de las deformaciones en una pieza dada. Para ello se marca la chapa a embutir con círculos, del mismo modo que se marcó la chapa para determinar las curvas limite de formalidad, pero esta vez, la pieza a embutir será la pieza real. Las deformaciones exigidas en las distintas partes de la pieza se miden y se marcan en la misma gráfica en que se marcó previamente la curva limite de formalidad, tal como se ilustra en las figuras 7 y 8 . Variaciones en el diseño de la misma pieza pueden ser evaluadas en cuanto al nivel de exigencia requerido, y eventualmente optimizar el espesor de la plancha requerido para embutir la pieza.

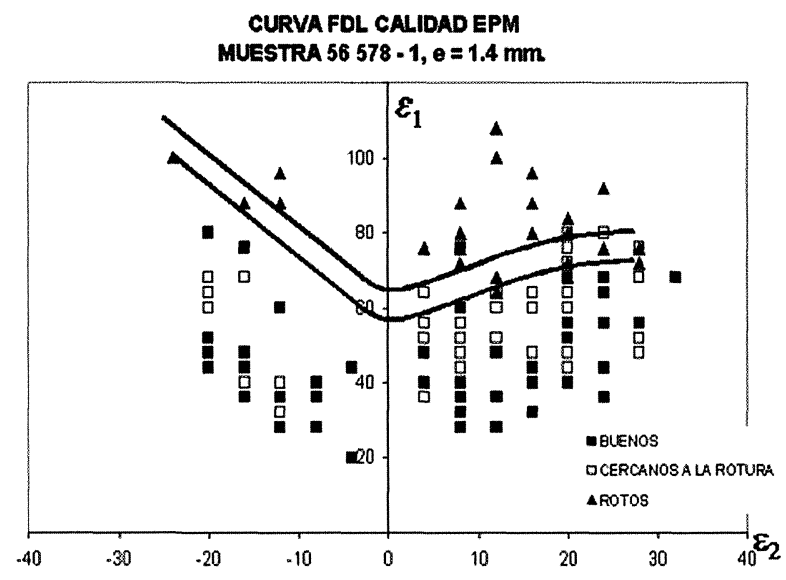

Figura 6. Curva límite de formabilidad de la chapa.

Figure 6. Forming limit curve of the steel sheet. 


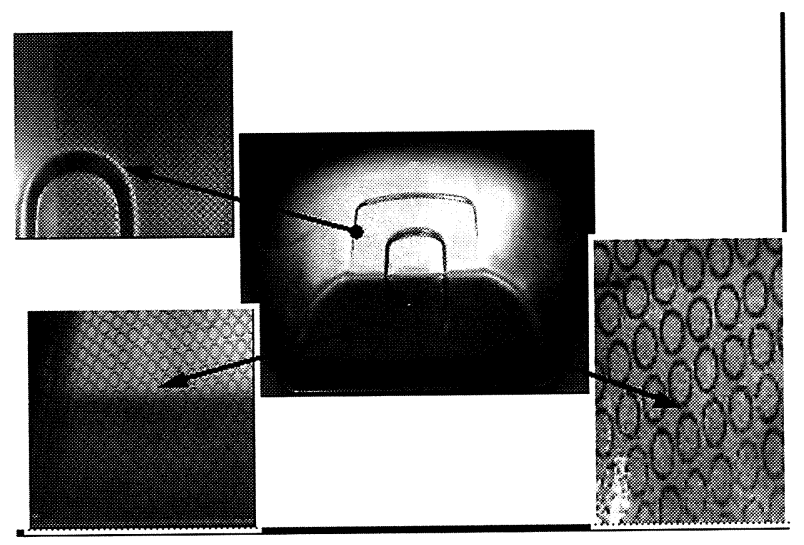

Figura 7. Determinación de deformaciones en la cubierta de una enceradora.

Figure 7. Deformation measurement on a polishing machine cover.

\section{REFERENCIAS}

[1] R.L. RicketT, S.H. Kalin y J.T. MaCKEnZIE JR., Metals Transactions, March (1949) 242-251.

[2] H. Pesenti, Trabajo de Titulación, Universidad de Atacama, Chile, 2002.

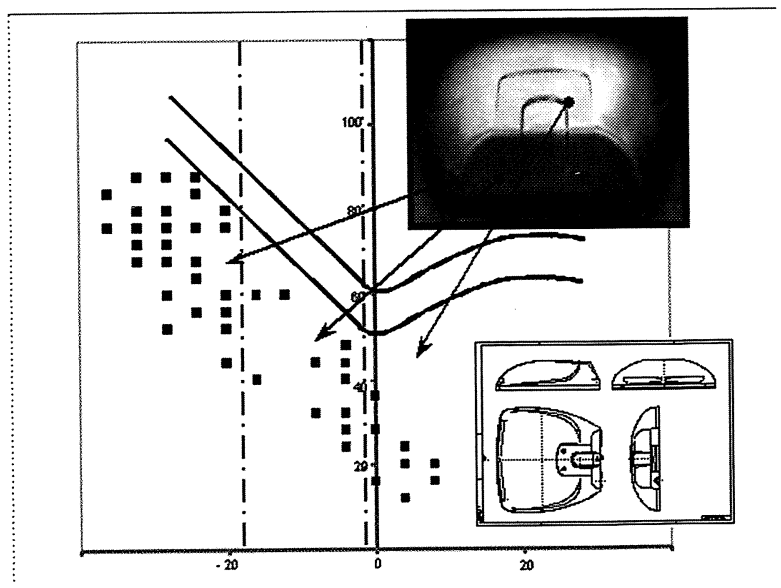

Figura 8. Trazado de las mediciones sobre la curva de deformación límite de la chapa.

Figure 8. Measurements drawn on the forming limit curve of the steel sheet.

[3] A. Artigas, M. Paez, D. Celentano y A. Monsalve, Proc. Conamet/Sam-Simposio Materia, Vol. I, 2002, pp. 341-346. 